\title{
The characteristics of the suicide attempter according to the onset time of the suicidal ideation
}

\author{
Kotaro Otsuka ${ }^{1,2^{*}}$, Hikaru Nakamura², Kaoru Kudo' , Jin Endo ${ }^{1}$, Katsumi Sanjo', Kentaro Fukumoto', \\ Katsuhito Hoshi ${ }^{1}$, Junko Yagi ${ }^{1}$ and Akio Sakai ${ }^{1}$
}

\begin{abstract}
Objective: To determine the timing of development of suicidal ideation and factors associated therewith in suicide attempters who required psychiatric emergency treatment.

Methods: Of a total of 2818 suicide attempters in Japan who presented to the primary or secondary emergency department of Iwate Medical University Hospital (hereinafter, referred to as our hospital) or Iwate Prefecture Advanced Emergency and Critical Care Center (hereinafter, referred to as the emergency center), an affiliated institution to our hospital, during the 12-year period from April 1, 2002-March 31, 2014, 2274 patients for whom the timing of development of suicidal ideation was identified were included in the study. The study subjects were classified into three groups according to the timing of development of suicide ideation: the "same-day" group, those who developed suicidal ideation and attempted suicide on the same day; the "short-term" group, those who developed suicidal ideation 2-7 days before attempting suicide; and the "long-term" group, those who developed suicidal ideation more than 7 days before attempting suicide. Factors associated with the development of suicidal ideation in each group were analyzed by a multiple logistic regression analysis with background factors, the diagnosis according to the ICD and the situations before and after the suicide attempt as explanatory variables.
\end{abstract}

Results: The same-day group was characterized by a high female ratio, high global functioning, low stress level, non-depressed status and a lack of seeking consultation. In contrast, the long-term group was characterized by low global functioning and a high stress level, suggesting that these patients exhibit consultation behavior, but have not received psychiatric services. In the short-term group, only male gender was identified as a significant factor.

Discussion: For those patients who developed suicidal ideation and attempted suicide on the same day, treatment strategies focusing on the acquisition of coping skills and stress management are recommended. For those with suicidal ideation lasting for more than a week or recurrent ideation, early detection and subsequent early treatment of such ideation are essential. In intermediate cases, treatment strategies that make the full use of mental health management in the workplace and gate-keeping are likely to be effective.

Keywords: Suicidal attempt, Suicide ideation, Brief psychiatric rating scale (BPRS), Global assessment scale (GAS), Life change units of Holmes social readjustment rating scale (LCU)

\footnotetext{
*Correspondence: kotaro29@df6.so-net.ne.jp

1 Department of Disaster and Community Psychiatry, Iwate Medical

University, Morioka, Japan

Full list of author information is available at the end of the article
} 


\section{Background}

A suicide attempt develops from suicidal ideation in the presence of complicating factors, such as anxiety, impulsivity and underlying psychiatric disorders. Zimri et al. have suggested that the trigger status of suicide is associated with factors such as the intensity of suicidal ideation, history of suicide attempt and current attempts [1], while others emphasize the importance of frantic hopelessness and loss of control over one's thinking as underlying triggers for suicidal ideation [2]. Some studies have also suggested that psychic pain, a condition characterized by increasing anxiety and restlessness, is experienced before the occurrence of serious suicide attempts [3], and that acute, intense negative feelings can also trigger suicide [4], [5]. However, suicidal ideation is in many cases a distinct entity, and do not always lead to suicide attempts.

Stress has long been identified as an important factor associated with suicide attempt. Mann et al. reported that the severity of current depression or psychosis cannot predict suicide attempt and proposed a stress-diathesis model composed of suicidal ideation, impulsivity, and feelings leading to suicide attempt [6]. While there are various known risk and protective factors associated with suicidal ideation and suicidal behaviors among young people, a population commonly affected by suicide attempts [7], stress has also been shown to have a role in the development of suicidal ideation in this population [8].

Interaction between stress factors and other pertinent factors may complicate stress management and consequently increase the risk of suicide attempt [9]. Controversy remains regarding the notion that stress is a predictor of suicidal tendency [10]. One study suggested that a high stress level does not predict the development of suicidal ideation because the relationship between depression and suicidal ideation is somewhat relaxed by a sudden stress event [11].

Based on the various perspectives, these facts indicate the importance of further analysis of suicidal ideation, which has a close connection to suicide attempt. Regarding the timing of development of suicidal ideation, the prevalence of suicidal ideation within the 2 weeks before suicide attempt is reported to be $4.8 \%$. Studies have identified the following factors to be associated with the development of suicidal ideation: male gender, high education level, smoking history, living alone, absence of religious behavior, financial strain, history of childhood abuse, family history of suicide, prior history of depression, current anxiety, coexistence of anxiety and depression, prior history of suicide attempt, indifference to one's own health, and use of antidepressants, as an indirect indicator of current depression, that not necessarily respond to the given drug [12]. To date, no report has fully explored the relationship between the timing of development of suicidal ideation and the characteristics and psychiatric profile of suicide attempters.

The crisis of the true suicide tends to be settled spontaneously in some period. Most patients can adapt themselves to the feelings that have finished becoming impoverished with the risk of the suicide within 24-48 h. The focus of our study is whether psychiatric staffs can management to suicidal ideation on the day and whether they can cope once a week in a generous limit of the short term treatment and what kind of characteristics the patients who no longer maintain suicidal ideation have [13]. Therefore, in this study, we classified suicide attempters who required psychiatric emergency treatment according to the timing of development of suicidal ideation, with the aim to determine the relationship between the time from the development of suicidal ideation to attempted suicide and the nature of suicide attempt.

\section{Methods}

A total of 18,639 patients presented to the primary or secondary emergency department of Iwate Medical University Hospital (hereinafter, referred to as our hospital) or the tertiary emergency department of a hospitalaffiliated Advanced Emergency and Critical Care Center (hereinafter, referred to as the emergency center) for psychiatric emergency services during a 12-year period between April 1, 2002 and March 31, 2014.

We use Silverman et al.s nomenclature for the study of suicidality, and therefore ascribe to their essential components of suicide and suicidal behaviors: suicide ideation, suicide and suicide attempts [14, 15]. Of the total, 2818 patients met the diagnostic criteria for suicide attempt by meeting any of the following criteria: 1 ) self-statement of suicide attempt, 2) presence of a suicide note or advance notice for suicide, 3 ) existence of a person witnessing the suicidal behavior, and 4) confirmation by judicial officials or autopsy (Kishi) [16]. Of these 2818 patients, 2274 patients for whom the time of development of suicidal ideation could be identified were included in the analysis (Table 1). Those whose death was confirmed after

Table 1 Timing of development of suicidal ideation

\begin{tabular}{lcr}
\hline & N & $\%$ \\
\hline Time from development of suicidal ideation to suicide attempt (days) \\
Same day & 1358 & 59.7 \\
$2-7$ & 492 & 21.6 \\
$>7$ & 424 & 18.6 \\
Total & 2274 & 100.0 \\
\hline
\end{tabular}


transportation to the emergency center and those who died within $24 \mathrm{~h}$ after admission were defined as suicides

The patients were classified into the following three groups according to the timing of development of suicidal ideation: the "same-day" group, consisting of those who developed suicidal ideation and attempted suicide on the same day; the "short-term" group, consisting of those who developed suicidal ideation 2-7 days before attempting suicide; and the "long-term" group, consisting of those who developed suicidal ideation more than 7 days before attempting suicide. Patients of each group were examined for gender, age, years of schooling, living status, work status, history of presentation to psychiatric services, consultation prior to suicide attempt, history of suicide-related behavior (lifetime and during the past year), motives and means of suicide attempt, Asukai grade [a grading system for the seriousness of attempted suicide based on its means and outcome; absolutely dangerous (AD) or relatively dangerous (RD) group (1995)] [17], Japan Coma Scale (JCS), diagnostic classification according to the Mental and behavioral disorders section of the International classification of diseases and related health problems, 10th edition (hereinafter, referred to as ICD-10) [18], and outcome of emergency treatment. Evaluable patients were also evaluated for psychiatric symptoms using the Oxford University Version of the brief psychiatric rating scale (BPRS) (translated into Japanese by Kitamura et al.) [19] and for overall psychiatric symptoms and daily life capacities using the global assessment scale (GAS) (translated into Japanese by Kitamura et al.) [20]. Because the large part of patients did not be able to diagnose the subgroup for an emergency stage, we investigated the classification of the evaluation to main cord (F1-F9, F99). In addition, the subjects' life events prior to suicide attempts, such as spouse's death and debts, were scored on a 0-100 scale using life change units (LCU) of the Holmes social readjustment rating scale [21].

Assessment of each investigation item and diagnoses were based on the information obtained from the patients and those surrounding them, such as family members, paramedics, hospital staff and primary care physicians, at the time of emergency psychiatric services. Suicides were evaluated based on the history of presentation to our department immediately before admission, or in the same manner as described above if they were able to talk at the time of presentation. Investigations and evaluations were conducted by 14 psychiatric emergency psychiatrists or psychiatrists on duty at our hospital, under the supervision of a senior psychiatrist (a designated psychiatrist).

SPSS $21.0 \mathrm{~J}$ for Windows was used for statistical processing. One-way analysis of variance was used for comparing the means of three groups (the "same-day", "short-term", and "long-term" groups), followed by the analysis of difference between two groups using the Bonferroni method. Chi-square test was used for the analysis of ratios among three groups. JCS and LCU data were analyzed using the Kruskal-Wallis test.

In order to identify factors associated with the timing of development of suicidal ideation, a multiple logistic analysis with the forced entry method was performed, with all investigation items (gender, age, years of schooling, living status, work status, diagnosis according to the ICD-10, history of presentation to psychiatric services, history of contact with outpatient psychiatric services, consultation prior to suicide attempt, history of suicide attempt during lifetime and the past year, LCU total score, motives of suicide attempt, first/return presentation, BPRS total score, means of suicide attempt, severity of physical condition (AD/RD group), GAS, and outcome) considered as explanatory variables and the "same-day", "short-term" and "long-term" groups as the dependent variables.

The significance level was set at $5 \%$ in all tests, with probabilities of significance presented numerically. Personally identifiable data were excluded from the analysis. Data were appropriately managed and processed to ensure the protection of personal information. This study was conducted with the approval of the ethics committees at Iwate Medical University School of Medicine.

\section{Results}

\section{Background}

The same-day group had the largest sample size, followed in order by the short-term and long-term groups. The number of women included was more than twice that of men. The female ratio was especially high in the same-day group compared to the other two groups. The mean age was the highest in the same-day group, followed in order by the short-term and long-term groups. The same-day group had lower percentages of patients who presented for the first time and those who received tertiary emergency services, and higher percentages of patients with prior history of contact with outpatient psychiatric services, history of suicide-related behavior during lifetime or during the past year, and no consultation prior to suicide attempt (Table 2).

\section{Diagnosis, psychiatric parameters, means of suicide attempt and outcome}

With respect to the ICD-10 classification, the distribution of mood disorder (F3), neurotic disorder (F4) and personality disorder (F6) significantly varied among three groups, with a higher percentage of F3 and lower percentages of F4 and F6 in the same-day group compared to the other groups. 
Table 2 Results of intergroup comparison

\begin{tabular}{|c|c|c|c|c|c|c|c|c|}
\hline \multirow[t]{2}{*}{ Factor } & \multirow{2}{*}{$\begin{array}{l}\text { Entire } \\
\text { population } \\
N=2274\end{array}$} & \multicolumn{3}{|c|}{$\begin{array}{l}\text { Time from development of suicidal ideation } \\
\text { to suicide attempt }\end{array}$} & \multirow[t]{2}{*}{$P$ value } & \multirow{2}{*}{$\begin{array}{l}\text { Multiple } \\
\text { comparison } \\
\text { and residual } \\
\text { analysis }\end{array}$} & \multirow[t]{2}{*}{$\begin{array}{l}\text { Analytical } \\
\text { method }\end{array}$} & \multirow[t]{2}{*}{$\begin{array}{l}\text { Unknown } \\
\mathrm{N}=544\end{array}$} \\
\hline & & $\begin{array}{l}\text { Same day } \\
N=1358\end{array}$ & $\begin{array}{l}\text { Short-term } \\
\text { (2-7 days) } \\
\mathrm{N}=492\end{array}$ & $\begin{array}{l}\text { Long-term } \\
\text { (>7 days) } \\
\mathrm{N}=424\end{array}$ & & & & \\
\hline \multicolumn{9}{|l|}{ Male } \\
\hline $\mathrm{N}$ & 657 & 308 & 188 & 161 & \multirow[t]{2}{*}{$<.001$} & \multirow[t]{2}{*}{ Shaded area } & \multirow[t]{2}{*}{ Chi-square } & 191 \\
\hline$\%$ & 28.9 & 22.7 & 38.2 & 38.0 & & & & 35.1 \\
\hline \multicolumn{9}{|l|}{ Age } \\
\hline Mean & 36.73 & 34.68 & 38.36 & 41.43 & \multirow[t]{2}{*}{$<.001$} & \multirow{2}{*}{$\begin{array}{l}\text { Significant difference } \\
\text { among } 3 \text { groups }\end{array}$} & \multirow{2}{*}{$\begin{array}{c}\text { Analysis of } \\
\text { variance }\end{array}$} & 40.53 \\
\hline SD & 16.29 & 15.11 & 16.62 & 18.27 & & & & 17.46 \\
\hline \multicolumn{9}{|c|}{ Presentation to tertiary services } \\
\hline $\mathrm{N}$ & 1421 & 802 & 331 & 288 & \multirow[t]{2}{*}{$<.001$} & \multirow[t]{2}{*}{ Shaded area } & \multirow[t]{2}{*}{ Chi-square } & 405 \\
\hline$\%$ & 62.5 & 59.1 & 67.3 & 67.9 & & & & 74.4 \\
\hline \multicolumn{9}{|c|}{ First-time presentation } \\
\hline $\mathrm{N}$ & 1181 & 629 & 279 & 273 & \multirow[t]{2}{*}{$<.001$} & \multirow[t]{2}{*}{ Shaded area } & & 297 \\
\hline$\%$ & 51.9 & 46.3 & 56.7 & 64.4 & & & & 54.6 \\
\hline Psychiat & tric diagnosis & & & & & & & \\
\hline Fo & & & & & & & & \\
\hline N & 47 & 29 & 12 & 6 & .533 & & & 17 \\
\hline$\%$ & 2.1 & 2.1 & 2.4 & 1.4 & & & & 3.1 \\
\hline F1 & & & & & & & & \\
\hline $\mathrm{N}$ & 68 & 49 & 9 & 10 & .097 & & & 12 \\
\hline$\%$ & 3.0 & 3.6 & 1.8 & 2.4 & & & & 2.2 \\
\hline $\mathrm{F} 2$ & & & & & & & & \\
\hline $\mathrm{N}$ & 310 & 186 & 70 & 54 & .802 & & & 71 \\
\hline$\%$ & 13.6 & 13.7 & 14.2 & 12.7 & & & & 13.1 \\
\hline F3 & & & & & & & & \\
\hline $\mathrm{N}$ & 837 & 400 & 210 & 227 & $<.001$ & Shaded area & & 235 \\
\hline$\%$ & 36.8 & 29.5 & 42.7 & 53.5 & & & & 43.2 \\
\hline F4 & & & & & & & & \\
\hline $\mathrm{N}$ & 696 & 470 & 132 & 94 & $<.001$ & Shaded area & & 110 \\
\hline$\%$ & 30.6 & 34.6 & 26.8 & 22.2 & & & & 20.2 \\
\hline F6 & & & & & & & & \\
\hline$N$ & 199 & 145 & 39 & 15 & $<.001$ & Shaded area & & 33 \\
\hline$\%$ & 8.8 & 10.7 & 7.9 & 3.5 & & & & 6.1 \\
\hline Other & & & & & & & & \\
\hline $\mathrm{N}$ & 117 & 79 & 20 & 18 & .208 & & & 67 \\
\hline$\%$ & 5.1 & 5.8 & 4.1 & 4.2 & & & & 12.3 \\
\hline Prior his & tory of suicide & & & & & & & \\
\hline During & $y$ the past year & & & & & & & \\
\hline $\mathrm{N}$ & 919 & 611 & 152 & 156 & $<.001$ & Shaded area & & 163 \\
\hline$\%$ & 40.4 & 45.0 & 30.9 & 36.8 & & & & 30.0 \\
\hline During & lifetime & & & & & & & \\
\hline$N$ & 1297 & 848 & 239 & 210 & $<.001$ & Shaded area & & 245 \\
\hline$\%$ & 57.0 & 62.4 & 48.6 & 49.5 & & & & 45.0 \\
\hline No cons & sultation prior $t$ & e attempt & & & & & & \\
\hline $\mathrm{N}$ & 1453 & 942 & 297 & 214 & $<.001$ & Shaded area & & \\
\hline$\%$ & 63.9 & 69.4 & 60.4 & 50.5 & & & & \\
\hline
\end{tabular}


Table 2 continued

\begin{tabular}{|c|c|c|c|c|c|c|c|c|}
\hline \multirow[t]{2}{*}{ Factor } & \multirow{2}{*}{$\begin{array}{l}\text { Entire } \\
\text { population } \\
\mathrm{N}=2274\end{array}$} & \multicolumn{3}{|c|}{$\begin{array}{l}\text { Time from development of suicidal ideation } \\
\text { to suicide attempt }\end{array}$} & \multirow[t]{2}{*}{$P$ value } & \multirow{2}{*}{$\begin{array}{l}\text { Multiple } \\
\text { comparison } \\
\text { and residual } \\
\text { analysis }\end{array}$} & \multirow[t]{2}{*}{$\begin{array}{l}\text { Analytical } \\
\text { method }\end{array}$} & \multirow[t]{2}{*}{$\begin{array}{l}\text { Unknown } \\
\mathrm{N}=544\end{array}$} \\
\hline & & $\begin{array}{l}\text { Same day } \\
N=1358\end{array}$ & $\begin{array}{l}\text { Short-term } \\
\text { (2-7 days) } \\
\mathrm{N}=492\end{array}$ & $\begin{array}{l}\text { Long-term } \\
\text { (>7 days) } \\
\mathrm{N}=424\end{array}$ & & & & \\
\hline \multicolumn{9}{|c|}{ Currently receiving outpatient psychiatric treatment } \\
\hline $\mathrm{N}$ & 1411 & 907 & 273 & 231 & $<.001$ & Shaded area & & \\
\hline$\%$ & 62.0 & 66.8 & 55.5 & 54.5 & & & & \\
\hline \multicolumn{9}{|c|}{ Means of suicide attempt } \\
\hline \multicolumn{9}{|l|}{ Drug } \\
\hline $\mathrm{N}$ & 1221 & 796 & 260 & 165 & $<.001$ & Shaded area & & 276 \\
\hline$\%$ & 53.7 & 58.6 & 52.8 & 38.9 & & & & 50.7 \\
\hline \multicolumn{9}{|c|}{ Poisoning } \\
\hline $\mathrm{N}$ & 137 & 65 & 34 & 38 & .004 & Shaded area & & 32 \\
\hline$\%$ & 6.0 & 4.8 & 6.9 & 9.0 & & & & 5.9 \\
\hline \multicolumn{9}{|c|}{ Inhaling gas } \\
\hline $\mathrm{N}$ & 77 & 27 & 26 & 24 & $<.001$ & Shaded area & & 34 \\
\hline$\%$ & 3.4 & 2.0 & 5.3 & 5.7 & & & & 6.2 \\
\hline \multicolumn{9}{|c|}{ Jumping from a tall building } \\
\hline $\mathrm{N}$ & 90 & 49 & 16 & 25 & .072 & & & 32 \\
\hline$\%$ & 4.0 & 3.6 & 3.3 & 5.9 & & & & 5.9 \\
\hline \multicolumn{9}{|c|}{ Jumping in front of a train } \\
\hline $\mathrm{N}$ & 9 & 6 & 1 & 2 & .742 & & & 1 \\
\hline$\%$ & .4 & .4 & .2 & .5 & & & & .2 \\
\hline \multicolumn{9}{|l|}{ Knife } \\
\hline N & 399 & 233 & 82 & 84 & .385 & & & 61 \\
\hline$\%$ & 17.5 & 17.2 & 16.7 & 19.8 & & & & 11.2 \\
\hline \multicolumn{9}{|c|}{ Self-burning } \\
\hline N & 22 & 10 & 7 & 5 & .364 & & & 19 \\
\hline$\%$ & 1.0 & .7 & 1.4 & 1.2 & & & & 3.5 \\
\hline \multicolumn{9}{|c|}{ Drowning oneself } \\
\hline $\mathrm{N}$ & 29 & 16 & 4 & 9 & .187 & & & 5 \\
\hline$\%$ & 1.3 & 1.2 & .8 & 2.1 & & & & .9 \\
\hline \multicolumn{9}{|c|}{ Hanging } \\
\hline N & 104 & 49 & 19 & 36 & $<.001$ & Shaded area & & 46 \\
\hline$\%$ & 4.6 & 3.6 & 3.9 & 8.5 & & & & 8.5 \\
\hline \multicolumn{9}{|c|}{ Combination } \\
\hline$N$ & 154 & 88 & 38 & 28 & .635 & & & 28 \\
\hline$\%$ & 6.8 & 6.5 & 7.7 & 6.6 & & & & 5.1 \\
\hline \multicolumn{9}{|l|}{ Other } \\
\hline$N$ & 18 & 10 & 4 & 4 & .914 & & & 2 \\
\hline$\%$ & .8 & .7 & .8 & .9 & & & & .4 \\
\hline \multicolumn{9}{|c|}{ Motives for suicide } \\
\hline \multicolumn{9}{|c|}{ Family problem } \\
\hline $\mathrm{N}$ & 502 & 336 & 102 & 64 & $<.001$ & Shaded area & & 108 \\
\hline$\%$ & 22.1 & 24.7 & 20.7 & 15.1 & & & & 19.9 \\
\hline Econor & mic difficulties & & & & & & & \\
\hline $\mathrm{N}$ & 119 & 47 & 40 & 32 & $<.001$ & Shaded area & & 24 \\
\hline$\%$ & 5.2 & 3.5 & 8.1 & 7.5 & & & & 4.4 \\
\hline
\end{tabular}


Table 2 continued

\begin{tabular}{|c|c|c|c|c|c|c|c|c|}
\hline \multirow[t]{2}{*}{ Factor } & \multirow{2}{*}{$\begin{array}{l}\text { Entire } \\
\text { population } \\
\mathrm{N}=2274\end{array}$} & \multicolumn{3}{|c|}{$\begin{array}{l}\text { Time from development of suicidal ideation } \\
\text { to suicide attempt }\end{array}$} & \multirow[t]{2}{*}{$P$ value } & \multirow{2}{*}{$\begin{array}{l}\text { Multiple } \\
\text { comparison } \\
\text { and residual } \\
\text { analysis }\end{array}$} & \multirow[t]{2}{*}{$\begin{array}{l}\text { Analytical } \\
\text { method }\end{array}$} & \multirow[t]{2}{*}{$\begin{array}{l}\text { Unknown } \\
\mathrm{N}=544\end{array}$} \\
\hline & & $\begin{array}{l}\text { Same day } \\
N=1358\end{array}$ & $\begin{array}{l}\text { Short-term } \\
\text { (2-7 days) } \\
\mathrm{N}=492\end{array}$ & $\begin{array}{l}\text { Long-term } \\
\text { (>7 days) } \\
\mathrm{N}=424\end{array}$ & & & & \\
\hline \multicolumn{9}{|c|}{ Pain of sickness } \\
\hline $\mathrm{N}$ & 243 & 112 & 60 & 71 & \multirow[t]{2}{*}{$<.001$} & \multirow[t]{2}{*}{ Shaded area } & & 69 \\
\hline$\%$ & 10.7 & 8.2 & 12.2 & 16.7 & & & & 12.7 \\
\hline \multicolumn{9}{|c|}{ Hallucinations/Delusion } \\
\hline $\mathrm{N}$ & 157 & 82 & 37 & 38 & \multirow[t]{2}{*}{.097} & & & 17 \\
\hline$\%$ & 6.9 & 6.0 & 7.5 & 9.0 & & & & 3.1 \\
\hline \multicolumn{9}{|c|}{ Work problem } \\
\hline $\mathrm{N}$ & 194 & 98 & 54 & 42 & \multirow[t]{2}{*}{.020} & \multirow[t]{2}{*}{ Shaded area } & & 34 \\
\hline$\%$ & 8.5 & 7.2 & 11.0 & 9.9 & & & & 6.2 \\
\hline \multicolumn{9}{|c|}{ Inter-personal relationship } \\
\hline $\mathrm{N}$ & 391 & 257 & 87 & 47 & \multirow[t]{2}{*}{.001} & \multirow[t]{2}{*}{ Shaded area } & & 60 \\
\hline$\%$ & 17.2 & 18.9 & 17.7 & 11.1 & & & & 11.0 \\
\hline \multicolumn{9}{|c|}{ School problem } \\
\hline $\mathrm{N}$ & 24 & 15 & 3 & 6 & \multirow[t]{2}{*}{.474} & & & 2 \\
\hline$\%$ & 1.1 & 1.1 & 6 & 1.4 & & & & .4 \\
\hline \multicolumn{9}{|l|}{ Other } \\
\hline $\mathrm{N}$ & 84 & 64 & 13 & 7 & \multirow[t]{2}{*}{.005} & \multirow[t]{2}{*}{ Shaded area } & & 12 \\
\hline$\%$ & 3.7 & 4.7 & 2.6 & 1.7 & & & & 2.2 \\
\hline Combir & ination & & & & & & & \\
\hline $\mathrm{N}$ & 431 & 247 & 86 & 98 & .050 & & & 97 \\
\hline$\%$ & 19.0 & 18.2 & 17.5 & 23.1 & & & & 17.8 \\
\hline Unknov & wn & & & & & & & \\
\hline N & 129 & 100 & 10 & 19 & $<.001$ & Shaded area & & 121 \\
\hline$\%$ & 5.7 & 7.4 & 2.0 & 4.5 & & & & 12.2 \\
\hline Living wi & vith someone & & & & & & & \\
\hline$N$ & 1826 & 1073 & 400 & 353 & .154 & & & 420 \\
\hline$\%$ & 80.3 & 79.0 & 81.3 & 83.3 & & & & 77.2 \\
\hline Years of $\mathrm{s}$ & schooling & & & & & & & \\
\hline Mean & 11.70 & 11.69 & 11.78 & 11.64 & .663 & & Analysis of & 11.63 \\
\hline SD & 2.35 & 2.19 & 2.39 & 2.75 & & & variance & 2.25 \\
\hline Being at & twork & & & & & & & \\
\hline $\mathrm{N}$ & 786 & 458 & 177 & 151 & .110 & & & 163 \\
\hline$\%$ & 34.6 & 33.7 & 36.0 & 35.6 & & & & 30.0 \\
\hline JCS & & & & & & & & \\
\hline Mean & 211.41 & 196.85 & 228.89 & 236.80 & .104 & & Kruskal-Wallis & S 103.85 \\
\hline SD & 373.87 & 362.48 & 386.16 & 392.83 & & & & 128.43 \\
\hline AD (Asuk & kai classificatic & & & & & & & \\
\hline N & 313 & 129 & 79 & 105 & $<.001$ & Shaded area & Chi-square & 120 \\
\hline$\%$ & 13.8 & 9.5 & 16.1 & 24.8 & & & & 22.1 \\
\hline BPRS & & & & & & & & \\
\hline Mean & 18.60 & 17.84 & 19.10 & 20.52 & $<.001$ & $\begin{array}{l}\text { Significant difference } \\
\text { between the same- } \\
\text { day group and the } \\
\text { other groups }\end{array}$ & $\begin{array}{c}\text { Analysis of } \\
\text { variance }\end{array}$ & 12.36 \\
\hline SD & 11.59 & 11.44 & 10.96 & 12.56 & & & & 9.80 \\
\hline
\end{tabular}


Table 2 continued

\begin{tabular}{|c|c|c|c|c|c|c|c|c|}
\hline \multirow[t]{2}{*}{ Factor } & \multirow{2}{*}{$\begin{array}{l}\text { Entire } \\
\text { population } \\
N=2274\end{array}$} & \multicolumn{3}{|c|}{$\begin{array}{l}\text { Time from development of suicidal ideation } \\
\text { to suicide attempt }\end{array}$} & \multirow[t]{2}{*}{$P$ value } & \multirow{2}{*}{$\begin{array}{l}\text { Multiple } \\
\text { comparison } \\
\text { and residual } \\
\text { analysis }\end{array}$} & \multirow[t]{2}{*}{$\begin{array}{l}\text { Analytical } \\
\text { method }\end{array}$} & \multirow[t]{2}{*}{$\begin{array}{l}\text { Unknown } \\
N=544\end{array}$} \\
\hline & & $\begin{array}{l}\text { Same day } \\
N=1358\end{array}$ & $\begin{array}{l}\text { Short-term } \\
\text { (2-7 days) } \\
N=492\end{array}$ & $\begin{array}{l}\text { Long-term } \\
\text { (>7 days) } \\
N=424\end{array}$ & & & & \\
\hline \multicolumn{9}{|l|}{ GAS } \\
\hline Mean & 34.74 & 37.16 & 32.99 & 29.08 & $<.001$ & $\begin{array}{l}\text { Significant difference } \\
\text { among } 3 \text { groups }\end{array}$ & & 24.81 \\
\hline SD & 19.26 & 19.80 & 17.77 & 17.78 & & & & 18.55 \\
\hline \multicolumn{9}{|l|}{ LCU } \\
\hline Mean & 41.70 & 31.852 & 29.225 & 39.378 & $<.001$ & $\begin{array}{l}\text { Significant difference } \\
\text { among } 3 \text { groups }\end{array}$ & Kruskal-Wallis & 52.84 \\
\hline SD & 33.40 & .981 & 1.476 & 2.056 & & & & 37.24 \\
\hline \multicolumn{9}{|c|}{ Outcome } \\
\hline \multicolumn{9}{|c|}{ Discharged home } \\
\hline N & 670 & 471 & 101 & 98 & $<.001$ & Shaded area & & 114 \\
\hline$\%$ & 30.2 & 35.3 & 21.0 & 24.3 & & & & 22.2 \\
\hline \multicolumn{9}{|c|}{ Admitted to emergency care center } \\
\hline $\mathrm{N}$ & 592 & 322 & 147 & 123 & .004 & Shaded area & Chi-square & 153 \\
\hline$\%$ & 26.7 & 24.1 & 30.6 & 30.4 & & & & 29.8 \\
\hline \multicolumn{9}{|c|}{ Admitted to psychiatric department } \\
\hline $\mathrm{N}$ & 937 & 532 & 225 & 180 & .016 & Shaded area & & 230 \\
\hline$\%$ & 42.2 & 39.9 & 46.9 & 44.6 & & & & 44.8 \\
\hline \multicolumn{9}{|c|}{ Suicide } \\
\hline $\mathrm{N}$ & 36 & 16 & 6 & 14 & .007 & Shaded area & & 52 \\
\hline$\%$ & 1.6 & 1.2 & 1.2 & 3.3 & & & & 9.6 \\
\hline
\end{tabular}

Regarding the general health performance (GAS average), significant differences were recognized among the three groups, with the long-term group having the highest level of seriousness, followed in order by the short-term and same-day groups. The score for psychiatric symptoms (BPRS total score) was higher in the same-day group compared to the long-term group while the score for life events (LCU average) was the highest in the short-term group, followed in order by the same-day and long-term groups. In terms of motives of suicide attempt, the same-day group had higher percentages of family problems, interpersonal relationship, other, and unknown motive, and a lower percentage of economic difficulties. Higher percentages of work problem and pain of sickness were observed in the short-term and long-term groups, respectively.

Among the means of suicide attempt, drug was the most common in all three groups and its percentage was the highest in the same-day group. The long-term group had higher percentages of poisoning and hanging compared to the other two groups. The percentage of inhaling gas was lower in the same-day group than the other groups. The percentage of patients classified in the AD group according to Asukai's criteria was the highest in the long-term group (24.8\%).
As for outcome, the percentage of patients discharged home was the highest in same-day group, and the percentages of patients admitted to the emergency center or psychiatric department in the same-day group were higher than the corresponding percentages in the short-term group. There were a total of 36 suicides $(1.6 \%)$ and the percentage of suicides was the highest in the long-term group (14 patients, $3.3 \%$ ) (Table 2).

\section{Logistic regression analysis}

Significant variables identified in the same-day group included male gender $(\mathrm{OR}=.682)$, mood disorder [F3] $(\mathrm{OR}=.536)$, consultation prior to suicide attempt $(\mathrm{OR}=.564)$, motives of economic difficulties $(\mathrm{OR}=.415)$, pain of sickness $(\mathrm{OR}=.453)$ and work problem $(\mathrm{OR}=.391)$, GAS score $(\mathrm{OR}=1.011)$, and LCU score $(\mathrm{OR}=.996)$. In the short-term group, male gender $(\mathrm{OR}=1.436)$ was identified as a significant variable. In the long-term group, first-time presentation $(\mathrm{OR}=1.486)$, consultation prior to suicide attempt $(\mathrm{OR}=1925)$, suicide attempt by drug $(\mathrm{OR}=.237)$, GAS score $(\mathrm{OR}=.986)$ and $\mathrm{LCU}(\mathrm{OR}=1.007)$ were identified as significant variables (Table 3 ). 
Table 3 The result of the logistic analysis of each group

\begin{tabular}{|c|c|c|c|c|c|c|c|c|c|c|c|c|}
\hline \multirow[t]{3}{*}{ Variables in the equation } & \multicolumn{4}{|c|}{ Same-day group $(\mathrm{N}=1358)$} & \multicolumn{4}{|c|}{ Short-term group $(\mathrm{N}=492)$} & \multicolumn{4}{|c|}{ Long-term group $(\mathrm{N}=424)$} \\
\hline & \multirow[t]{2}{*}{ OR } & \multicolumn{3}{|c|}{$95 \% \mathrm{Cl}$} & \multirow[t]{2}{*}{ OR } & \multicolumn{3}{|c|}{$95 \% \mathrm{Cl}$} & \multirow[t]{2}{*}{ OR } & \multicolumn{3}{|c|}{$95 \% \mathrm{Cl}$} \\
\hline & & Lower & Upper & P value & & Lower & Upper & P value & & Lower & Upper & $P$ value \\
\hline Male (female) & .682 & .521 & .894 & .006 & 1.436 & 1.061 & 1.944 & .019 & 1.154 & .833 & 1.599 & .389 \\
\hline Age & .996 & .988 & 1.005 & .375 & 1.000 & .990 & 1.009 & .941 & 1.005 & .995 & 1.016 & .311 \\
\hline Tertiary (primary/secondary) & .921 & .699 & 1.213 & .557 & 1.035 & .752 & 1.425 & .833 & 1.090 & .769 & 1.543 & .629 \\
\hline First presentation (return) & .816 & .634 & 1.050 & .114 & .933 & .697 & 1.248 & 639 & 1.486 & 1.085 & 2.036 & .014 \\
\hline \multicolumn{13}{|l|}{ Diagnosis } \\
\hline Fo & .898 & .361 & 2.233 & .817 & 1.906 & .707 & 5.140 & .202 & .459 & .126 & 1.678 & .239 \\
\hline F1 & 1.598 & .674 & 3.787 & .287 & .527 & .177 & 1.569 & .250 & .953 & .329 & 2.756 & .929 \\
\hline $\mathrm{F} 2$ & .766 & .405 & 1.450 & .413 & 1.515 & .712 & 3.224 & .281 & .896 & .396 & 2.026 & .792 \\
\hline F3 & .536 & .302 & .950 & .033 & 1.242 & .625 & 2.467 & .536 & 1.853 & .905 & 3.793 & .092 \\
\hline $\mathrm{F} 4$ & .904 & .511 & 1.601 & .729 & 1.034 & .520 & 2.056 & .924 & 1.071 & .517 & 2.218 & .854 \\
\hline F6 & .969 & .499 & 1.881 & .926 & 1.641 & .757 & 3.558 & .210 & .485 & .190 & 1.239 & .131 \\
\hline \multicolumn{13}{|l|}{ Prior history of suicide attempt } \\
\hline History of suicide attempt during the past year & $r 1.212$ & .868 & 1.691 & .259 & .593 & .404 & .870 & .008 & 1.287 & .837 & 1.981 & .250 \\
\hline History of suicide attempt during lifetime & 1.043 & .757 & 1.437 & .799 & .982 & .689 & 1.401 & .922 & .925 & .615 & 1.392 & .708 \\
\hline $\begin{array}{l}\text { Consultation prior to suicide attempt (no } \\
\text { consultation) }\end{array}$ & .564 & .449 & .707 & .000 & 1.198 & .924 & 1.552 & .172 & 1.925 & 1.462 & 2.535 & .000 \\
\hline $\begin{array}{l}\text { Prior contact with outpatient psychiatric service } \\
\text { (no prior contact) }\end{array}$ & 1.157 & .890 & 1.503 & .275 & .830 & .616 & 1.120 & .223 & .995 & .722 & 1.372 & .976 \\
\hline \multicolumn{13}{|l|}{ Means of suicide attempt } \\
\hline Drug & 3.005 & .603 & 14.970 & .179 & 1.131 & .127 & 10.049 & .912 & .237 & .047 & 1.206 & .083 \\
\hline Poisoning & 2.336 & .441 & 12.361 & .318 & 1.141 & .121 & 10.716 & .908 & .350 & .064 & 1.928 & .228 \\
\hline Inhaling gas & 1.838 & .320 & 10.552 & .495 & 1.244 & .126 & 12.270 & .852 & .366 & .062 & 2.147 & .266 \\
\hline Jumping from a tall building & 2.572 & .461 & 14.356 & .281 & .684 & .067 & 6.985 & .749 & .458 & .079 & 2.662 & .385 \\
\hline Jumping in front of a train & 8.346 & .547 & 127.353 & .127 & .781 & .035 & 17.325 & .876 & .000 & .000 & & .999 \\
\hline Knife & 2.420 & .479 & 12.233 & .285 & .924 & .102 & 8.363 & .944 & .396 & .077 & 2.046 & .269 \\
\hline Self-burning & 5.057 & .728 & 35.130 & .101 & .755 & .062 & 9.189 & .826 & .194 & .023 & 1.620 & .130 \\
\hline Drowning oneself & 1.340 & .196 & 9.144 & .765 & .682 & .054 & 8.595 & .767 & .972 & .142 & 6.644 & .977 \\
\hline Hanging & 3.025 & .547 & 16.736 & .205 & .604 & .060 & 6.088 & .669 & .408 & .072 & 2.322 & .313 \\
\hline Combination & 1.918 & .371 & 9.926 & .437 & 1.641 & .179 & 15.054 & .661 & .298 & .055 & 1.605 & .159 \\
\hline Other & 2.874 & .391 & 21.104 & .299 & 1.161 & .090 & 14.959 & .909 & .265 & .028 & 2.529 & .248 \\
\hline \multicolumn{13}{|l|}{ Motives for suicide attempt outcome } \\
\hline Family problem & .826 & .417 & 1.635 & .583 & 1.221 & .552 & 2.702 & .623 & 1.144 & .440 & 2.977 & .782 \\
\hline Economic difficulties & .415 & .187 & .921 & .031 & 1.659 & .678 & 4.063 & .268 & 2.095 & .737 & 5.952 & .165 \\
\hline Pain of sickness & .453 & .215 & .958 & .038 & 1.471 & .621 & 3.487 & .381 & 2.234 & .826 & 6.039 & .113 \\
\hline Hallucinations/Delusion & .580 & .265 & 1.271 & .174 & 1.214 & .489 & 3.015 & .676 & 2.139 & .744 & 6.144 & .158 \\
\hline Work problem & .391 & .186 & .825 & .014 & 1.729 & .739 & 4.046 & .207 & 2.344 & .862 & 6.372 & .095 \\
\hline Interpersonal relationship & .571 & .285 & 1.144 & .114 & 1.524 & .681 & 3.411 & .305 & 1.526 & .575 & 4.049 & .396 \\
\hline Combined motives & .588 & .297 & 1.163 & .127 & 1.290 & .582 & 2.857 & .531 & 1.844 & .718 & 4.740 & .204 \\
\hline School problem & .555 & .156 & 1.977 & .363 & .718 & .132 & 3.906 & .701 & 3.616 & .787 & 16.613 & .098 \\
\hline Unknown motive & 1.577 & .665 & 3.741 & .301 & .418 & .136 & 1.286 & .128 & 1.112 & .354 & 3.495 & .856 \\
\hline Living with someone (alone) & .776 & .579 & 1.040 & .089 & 1.265 & .898 & 1.782 & .180 & 1.131 & .788 & 1.623 & .505 \\
\hline Years of schooling & .999 & .952 & 1.049 & .973 & .991 & .938 & 1.048 & .756 & 1.012 & .954 & 1.074 & .684 \\
\hline Being at work (not working) & 1.074 & .837 & 1.379 & .573 & .951 & .715 & 1.266 & .731 & .949 & .697 & 1.291 & .738 \\
\hline JCS & 1.000 & .999 & 1.000 & .378 & 1.000 & 1.000 & 1.000 & .935 & 1.000 & 1.000 & 1.001 & .375 \\
\hline AD group (RD) & .707 & .484 & 1.033 & .073 & 1.073 & .707 & 1.629 & .741 & 1.406 & .918 & 2.152 & .117 \\
\hline Total BPRS & .991 & .979 & 1.002 & .104 & 1.001 & .988 & 1.015 & .830 & 1.012 & .999 & 1.026 & .076 \\
\hline GAS & 1.011 & 1.004 & 1.017 & .001 & .997 & .990 & 1.004 & .450 & .986 & .978 & .994 & .001 \\
\hline
\end{tabular}


Table 3 continued

\begin{tabular}{|c|c|c|c|c|c|c|c|c|c|c|c|c|}
\hline \multirow[t]{3}{*}{ Variables in the equation } & \multicolumn{4}{|c|}{ Same-day group $(\mathrm{N}=1358)$} & \multicolumn{4}{|c|}{ Short-term group $(\mathrm{N}=492)$} & \multicolumn{4}{|c|}{ Long-term group $(\mathrm{N}=424)$} \\
\hline & \multirow[t]{2}{*}{ OR } & \multicolumn{3}{|c|}{$95 \% \mathrm{Cl}$} & \multirow[t]{2}{*}{ OR } & \multicolumn{3}{|c|}{$95 \% \mathrm{Cl}$} & \multirow[t]{2}{*}{ OR } & \multicolumn{3}{|l|}{$95 \% \mathrm{Cl}$} \\
\hline & & Lower & Upper & $P$ value & & Lower & Upper & $P$ value & & Lower & Upper & $P$ value \\
\hline LCU & .996 & .993 & 1.000 & .047 & .998 & .994 & 1.002 & .363 & 1.007 & 1.003 & 1.011 & .001 \\
\hline \multicolumn{13}{|l|}{ Outcome } \\
\hline Discharged home & 1.234 & .230 & 6.608 & .806 & .628 & .113 & 3.480 & .594 & 1.351 & .134 & 13.674 & .799 \\
\hline Admitted to emergency center & 1.361 & .253 & 7.334 & .720 & .711 & .128 & 3.943 & .696 & 1.023 & .102 & 10.303 & .984 \\
\hline Admitted to psychiatric department & .942 & .177 & 5.014 & .944 & .843 & .154 & 4.621 & .844 & 1.476 & .148 & 14.774 & .740 \\
\hline
\end{tabular}

Variables included: gender, age, primary/secondary/tertiary services, first/return presentation, f0, f1, f2, f3, f4, f6, with or without SMI 1 year for presentation/absence and logistic analysis, with or without history of SMV for logistic analysis, with or without prior consultation for logistic analysis, contact with outpatient psychiatric analysis for logistic analysis, drug, poisoning, inhaling gas, jumping from a tall building, jumping in front of a train, knife, self-burning, drowning oneself, hanging, combination, other, family problem, economic difficulties, pain of sickness, hallucination/delusion, work problem, interpersonal relationship, combined motives, school problem, unknown motive, living with someone or alone, years of schooling, work status, JCS, ADRD, total BPRS, GAS, LCU, discharged home or not, admitted to emergency center or not, admitted to psychiatric department or not

\section{Discussion}

Variables identified by a multivariate analysis as differentiating the three groups were gender, first/return presentation, F3 component of the ICD-10, consultation prior to suicide attempt, drug as a means of suicide attempt, economic difficulties, pain of sickness and work problem as motives for suicide attempt, LCU, and GAS.

\section{Same-day group}

Suicidal ideation that occurred on the day of suicide attempt was significantly associated with female gender, being not depressed, no consultation prior to suicide attempt, suicide attempt by means of drug, suicide attempt not motivated by economic difficulties, pain of sickness or work problem, low LCU, and high GAS. Global function was rated high, suggesting the involvement of vulnerability in coping with stress, with which even life events of low stress levels can lead to suicidal ideation.

Not all suicide-related behaviors are intended for death; some behaviors serve as a subconscious signal for help. Such help-seeking behaviors are particularly notable in women, and are grouped under the concept of "parasuicides" [22, 23]. In the same-day group, it appeared that help-seeking behavior that occurred before suicide attempt did not directly lead to consultation or treatment and did not prevent suicide attempt. Regarding life events, suicide attempts in young people are likely to be triggered by interpersonal relationship-related events, and this risk appears to be higher for women than for men [24, 25]. In order to reduce stress, it is important to maintain good quality of life and integrate problem coping and solving [26]. Ibrahim et al. have suggested that coping skills required for pain management should be reinforced to reduce depression and suicide among young people [26]. In order to prevent the development of suicidal ideation followed by suicide attempt on the same day, supports are needed to facilitate the acquisition of stress management and stress coping skills and the control of mental conditions that can easily lead to direct actions, such as anxiety and impulsivity.

A higher percentage of patients in the same-day group had history of prior suicide attempt and were on outpatient psychiatric treatment compared to the other groups. It should be noted that parasuicide cases who do not have physically serious conditions and have exhibited suiciderelated behaviors several times, and those whose suicidal feelings were temporarily weakened after an attempt due to its cathartic effect [27], are very likely to repeat their attempts, finally with a higher rate of fatality $[28,29,30]$. Joiner et al. have identified "acquired suicide potential" as a key risk factor for suicide [31]. This represents an insensitivity to physical pain and lack of fear for suicide acquired through repeated suicide-related behaviors and other similar experiences. These experiences appear to serve as a type of "rehearsal" for suicide and reduce the threshold for suicidal behaviors [32]. Even if a patient is judged to be safe enough to return home after outpatient treatment, it is necessary to determine the process by which he/she came to try to kill him/herself, and to provide careful treatment, such as the introduction of proper psychotherapy or encouragement to visit a psychiatrist in the future. A relatively short interval from the development of suicidal ideation to occurrence of suicide attempt also suggests the need for the proactive use of psychiatric emergency and hot-line call services and providing support to the patient's family and the surrounding people.

\section{Short-term group}

In the short-term group, only male gender was identified as a significant variable associated with the development of suicide ideation. It is more likely that, compared to 
women, men do not consult with the people around them prior to suicide attempt and often refuse to see a psychiatrist, even if the people around them notice changes and encourage them to see a psychiatrist. It has also been suggested that men tend to have too much stress themselves without consulting the people around them, and develop psychological tunnel vision [33]. The first thing to be done is to vigorously promote mental health management in the workplace to prevent the development of suicidal ideation and the occurrence of suicide attempt. Given an interval of 1 week from suicidal ideation to attempt, strategies that make the full use of gate-keeping will be effective in preventing these events.

Specifically, it is important to prevent suicide attempts that can occur within a limited time frame by assessing items identified as significant factors in the same-day and long-term groups, such as a history of presentation to psychiatric services, opportunity for consultation prior to suicide attempt, global seriousness, and stressful life events. For example, a treatment strategy consisting of a combination of various types of care has been shown to reduce the severity of depressive symptoms and the development of suicidal ideation among elderly depressed patients [30]. A substantial effect is likely to be achieved by a comprehensive treatment strategy consisting of counseling services that address stress coping, social work services that support problem solving, and drug therapy that includes impulsivity control in its scope.

\section{Long-term group}

In the long-term group, which consisted of those who attempted suicide more than a week after the development of suicidal ideation, significant factors associated with the development of suicidal ideation included firsttime presentation, consultation prior to suicide attempt, no use of a drug as a means of suicide attempt, life events associated with a high stress level, and severely impaired global functioning.

This group is characterized by no previous contact with psychiatric services even after consultation with surrounding people. It is well known that suicides tend to have no previous contact with psychiatric services before committing suicidal behavior [34]. In these cases, where serious untreated episodes are likely to be present, the condition itself may direct the patient to use a serious means of suicide other than drugs, or the presence of untreated episodes itself may prevent them from selecting drugs as a means of suicide attempt. Our previous study has demonstrated that lower GAS is associated with an increased frequency of suicide attempts by dangerous means and that GAS predicts the occurrence of dangerous suicide attempt, regardless of gender, age, or even disease entity [35]. Although no correlation to the emergence of AD group was identified in the present study, it should be noted that the selection of a non-drug means of suicide attempt may lead to serious consequences. Although no significant difference was found in the distribution of outcomes among groups, the longterm group had a slightly higher percentage of suicides (3.3\%) compared to the other groups.

Disorders characterized by impulse-control and anxiety may be the most important in predicting the transition from suicidal ideation to suicide attempt [36]. Having not received any treatment indicates that these problems also remain unsolved. Monitoring of these risk factors is essential when conducting risk assessment [37]. Monitoring the impact of early-life and recent events in vulnerable individuals should be part of risk assessment and treatment [37]. In conclusion, for patients with persistent suicidal ideation, the problem-solving approaches should be selected based on a focus on life events associated with high stress levels. These patients should also be introduced to psychiatric treatment through procedures such as screening and gate-keeping.

\section{Limitations and future research}

Although this was a large scale study involving more than 2000 suicide attempters, the investigation was performed at a single institution. The study population also included 544 patients with unknown timing of development of suicidal ideation. This group of patients (the "unknown" group) had a lower female ratio compared to the sameday group, was older compared to the short-term group, and younger than the long-term group. The "unknown" group also had relatively higher percentages of patients with a history of contact with outpatient psychiatric services $(63.9 \%)$, and patients with a prior history of suicide attempt during the past year (>40\%) or lifetime (57 \%). This group also included a relatively high percentage of patients presenting to tertiary emergency services, including those from whom detailed descriptions of suicidal ideation could not be obtained after awakening from unconsciousness, and those who were not sure of the precise time of onset of suicidal ideation.

\section{Conclusion}

The present study revealed varying characteristics of suicide attempters depending on the timing of onset of suicidal ideation. The group of patients who attempted suicide within $24 \mathrm{~h}$ after the development of suicidal ideation was characterized by a higher female ratio, lack of consultation behavior, life events associated with a low stress level, and high global functioning. These patients are likely to be effectively treated by treatment strategies focusing on the acquisition of coping skills and stress 
management. The long-term group was characterized by life events associated with high stress levels, severely impaired global functioning, and no previous contact with psychiatric services even after consultation with the surrounding people. For these patients with persistent or recurrent suicidal ideation, early control of symptoms and the utilization of social resources with problem-solving intention are necessary. For the short-term group, suicide risk should be reduced by taking into account the factors which were identified as being significantly associated with the development of suicidal ideation in the same-day and long-term groups.

The present results did not provide insight into the relationship between the timing of development of suicidal ideation and outcome. No significant difference was found in terms of diagnostic category, except for diagnoses other than depression in the same-day group, suggesting a greater role of overall psychiatric severity, rather than specific psychiatric conditions, in predicting the outcome of suicide attempts. Future tasks include a more detailed assessment of suicide risk, taking into account factors in addition to the timing of onset of suicidal ideation.

\section{Authors' contributions}

$\mathrm{KO}$ wrote the paper. AS supervised and wrote the paper. HN participated in the design of the study and performed the statistical analysis. KK, JE, KS, KF, KH, and JY participated in the study as a whole and commented on the manuscript. AS conceived the study, and participated in its design and coordination. All authors read and approved the final manuscript.

\section{Author details}

${ }^{1}$ Department of Disaster and Community Psychiatry, Iwate Medical University, Morioka, Japan. ${ }^{2}$ Department of Neuropsychiatry, Iwate Medical University, Morioka, Japan.

\section{Acknowledgements}

We would like to express our heartfelt gratitude to the medical staff at the Department of Emergency Medicine and the Department of Neuropsychiatry, Iwate Medical University, for their cooperation in conducting this study.

\section{Competing interests}

This study was supported by research grant from Medical Service Bureau of Iwate Prefecture.

Received: 28 August 2015 Accepted: 7 December 2015

Published online: 30 December 2015

\section{References}

1. Yaseen ZS, Gilmer E, Modi J, Cohen L, Galynke II. Emergency room validation of the revised suicide trigger scale (STS-3): a measure of a hypothesized suicide trigger State. PLOS ONE. 2012;7(9):e45157. doi:10.1371/ journal.pone.0045157PMCID:PMC3443232 (Published online)

2. Galynker I, Yaseen Z, Briggs J. Assessing risk for imminent suicide. Psychiatr Ann. 2014;44(9):431-6.

3. Fawcett J. Depressive Disorders. In: Simon RI, Hales RE, editors. Textbook of suicide assessment and management. New York: Am Psychiatr Publ; 2006. p. 1-24.
4. Hendin H, Al Jurdi RK, Houck PR, Hughes S, Turner JB. Role of intense affects in predicting short-term risk for suicidal behavior: a prospective study. J Nerv Ment Dis. 2010;198(3):220-5.

5. Hendin $\mathrm{H}$, Maltsberger JT, Szanto K. The role of intense affective states in signaling a suicide crisis. J Nerv Ment Dis. 2007;195(5):363-8.

6. Mann JJ, Waternaux C, Haas GL, Malone KM. Toward a clinical model of suicidal behavior in psychiatric patients. Am J Psychiatry. 1999;156(2):181-9.

7. Gutierrez PM, Osman A. Adolescent suicide: an integrated approach to the assessment of risk and protective factors. DeKalb: Northern Illinois University Press; 2008.

8. Overholser JC. Predisposing factors in suicide attempts: life stressors. In: Spirito A, Overholser J, editors. Evaluating and treating adolescent suicide attempters: from research to practice. New York: Academic Press; 2003. p. 42-9.

9. Lee SI, Jung H. Psychosocial risk factors for suicide. Psychiatr Invest. 2006:3:15-22

10. Zhang XY, Wang HP, Xia Y, Liu XH, Jung EJ. Stress, coping and suicide ideation in Chinese college students. J Adolesc. 2011;35:1-8.

11. Yen S, Shea MT, Pagno M, Sanislow CA, Grilo CM. Axis I and axis II disorders as predictors of prospective suicide attempts: findings from the collaborative longitudinal personality disorders study. J Abnorm Psychol. 2003;112:375-81.

12. Almeida OP, Draper B, Snowdon J, Lautenschlager NT, Pirkis J, Byrne G, Sim M, Stocks N, Flicker L, Pfaff JJ. Factors associated with suicidal thoughts in a large community study of older adults. Br J Psychiatry. 2012;201:466-72. doi:10.1192/bjp.bp.112.110130.

13. Chiles JA, Strosahl KD. Clinical manual for assessment and treatment of suicidal patients. Washington DC and London, UK: American Psychiatric Publishing Inc; 2005.

14. Silverman MM, Berman AL, Sanddal ND, O'carroll PW, Joiner TE. Rebuilding the tower of Babel: a revised nomenclature for the study of suicide and suicidal behaviors. Part 1: background, rationale, and methodology. Suicide Life Threat Behav. 2007;37(3):248-63.

15. Silverman MM, Berman AL, Sanddal ND, O'carroll PW, Joiner TE. Rebuilding the tower of Babel: a revised nomenclature for the study of suicide and suicidal behaviors. Part 2: suicide-related ideations, communications, and behaviors. Suicide Life Threat Behav. 2007;37(3):264-77.

16. Kishi Y, Hosaka T, Kurosawa H. Proposal for establishment of suicide statistics at emergency care centers. J Clin Exp Med. 2000;194:555-9.

17. Asukai N. Suicide and mental disorders. Psychiatry Clin Neurosci. 1995:49:91-7

18. World Health Organization. The ICD-10 classification of mental and behavioural disorders: clinical descriptions and diagnostic guidelinesversion 1. Tokyo: Igaku-Shoin; 1993

19. Kitamura T, Machizawa S, Maruyama S, et al. Retest reliability of the Oxford University version of the brief psychiatric rating scale (BPRS): a preliminary investigation of a multi-center study sponsored by the National Institute of Mental Health. J Natl Inst Ment Health. 1986:32:1-5.

20. Endicott J, Spitzer RL, Fleiss JL, et al. The global assessment scale: a procedure for measuring overall severity of psychiatric disturbances. Arch Gen Psychiatry. 1976;33:766-71.

21. Holmes TH. Life situations, emotions, and disease. Psychosomatics. 1978;19:747-54

22. Suzuki $H$. The realities of attempted suicides at the emergency service of a university hospital. J Clin Exp Med. 2000;194:541-4.

23. Okamoto K, Sakata Y, Shimokawa K, et al. Sex difference in help-seeking behaviors. J J Psychosom Med. 2002;42(10):689.

24. Bridge JA, Goldestein TR, Brent DA. Adolescent suicide and suicide behavior. J Child Psychol Psychiatry. 2006;46:372-94.

25. Hawton K, van Heeringen K. Suicide. Lancet. 2009;373:1372-81.

26. Ibrahim N, Amit N, Suen MW. Psychological factors as predictors of suicidal ideation among adolescents in Malaysia. PLOS ONE. 2014;9(10):e110670. doi:10.1371/journal.pone.0110670 (eCollection 2014).

27. Matsuishi K, Kitamura N, Sato M, et al. Change of suicidal ideation induced by suicide attempt. Psychiatry Clin Neurosci. 2005;59:599-604.

28. Jenkins GR, Hale R, Papanastassiou M, et al. Suicide rate 22 years after parasuicide: cohort study. BMJ. 2002;325:1155. 
29. Suokas J, Suominen K, Isometsä E, et al. Long-term risk factors for suicide mortality after attempted suicide-findings of a 14-year follow-up study. Acta Psychiatr Scand. 2001;104:117-21.

30. Suominen K, Isometsä E, Suokas J, et al. Completed suicide after a suicide attempt: a 37-year follow-up study. Am J Psychiatry. 2004;161:562-3.

31. Joiner TE Jr, Van Orden KA, Witte TK, et al. The interpersonal theory of suicide: guidance for working with suicidal clients. Washington: American Psychological Association; 2009.

32. Schnaideman ES. Suicide as Psychache: a clinical approach to selfdestructive behavior. Kongo Shuppan: Tokyo; 2005. p. 37.

33. Bruce ML, Ten Have TR, Reynolds CF, Katz II, Schulberg HC, Mulsant BH, et al. Reducing suicidal ideation and depressive symptoms in depressed older primary care patients: a randomized controlled trial. JAMA. 2004;291:1081-91.

34. Vassilas C, Morgan $\mathrm{H}$. Elderly suicides' contact with their general practitioner before death. Int J Geriatr Psychiatry. 1994;9:1008-9.
35. Endo J, Otsuka K, Yoshida T, Nakamura H, Yambe T, Isono H, Chida F. On factors related to risk to life in attempted suicides: comparison between absolutely and relatively dangerous suicides in emergency and critical care center. J J Assoc Emerg Psychiatry. 2009;12:60-73.

36. Borges G, Nock MK, Abad JMH, Hwang I, Sampson NA, Alonso J, Andrade $\mathrm{LH}$, Angermeyer MC, Beautrais A, Bromet E, Bruffaerts R, de Girolamo G, Florescu S, Gureje O, Hu C, Karam EG, Kovess-Masfety V, Lee S, Levinson D, Medina-Mora ME, Ormel J, Posada-Villa J, Sagar R, Tomov T, Uda H, Williams DR, Kessler RC. Twelve Month prevalence of and risk factors for suicide attempts in the WHO World Mental Health Surveys. J Clin Psychiatry. 2010;71(12):1617-28.

37. Pompili M, Innamorati M, Szanto K, Di Vittorio C, Conwell Y, Lester D, Tatarelli $R$, Girardi P, Amore M. Life events as precipitants of suicide attempts among first-time suicide attempters, repeaters, and non-attempters. Psychiatry Res. 2011;186(2-3):300-5. doi:10.1016/j.psychres.2010.09.003.

\section{Submit your next manuscript to BioMed Central and we will help you at every step:}

- We accept pre-submission inquiries

- Our selector tool helps you to find the most relevant journal

- We provide round the clock customer support

- Convenient online submission

- Thorough peer review

- Inclusion in PubMed and all major indexing services

- Maximum visibility for your research

Submit your manuscript at www.biomedcentral.com/submit
() Biomed Central 